\title{
Dynamic compression response of self-reinforced poly(ethylene terephthalate) composites and corrugated sandwich cores \\ C. Schneider ${ }^{1,1}$, S. Kazemahvazi ${ }^{2}$, D. Zenkert ${ }^{1}$ and V. S. Deshpande ${ }^{2}$ \\ ${ }^{1}$ KTH, Department of Aeronautical and Vehicle Engineering, Stockholm, Sweden \\ ${ }^{2}$ Department of Engineering, University of Cambridge, Cambridge, CB2 1PZ, UK
}

\begin{abstract}
A novel manufacturing route for fully recyclable corrugated sandwich structures made from self-reinforced poly(ethylene terephthalate) SrPET composites is developed. The dynamic compression properties of the SrPET material and the out-of-plane compression properties of the sandwich core structure are investigated over a strain rate range $10^{-4} \mathrm{~s}^{-1}$ to $10^{3} \mathrm{~s}^{-1}$. Although the SrPET material shows limited rate dependence, the corrugated core structures show significant rate dependence mainly attributed to micro-inertial stabilisation of the core struts and increased plastic tangent stiffness of the SrPET material. The corrugated SrPET cores have similar quasi-static performance as commercial polymeric foams but the SrPET cores have superior dynamic compression properties.
\end{abstract}

Keywords: B.Mechanical Properties; B. Plastic deformation; A. Polymer (textile) fibre; Ductile composites

\section{Introduction}

The high specific stiffness and strength of composite materials (on a weight basis), such as carbon fibre reinforced polymers, makes these materials suitable for a range of lightweight applications. However, these traditional composites have some critical shortcomings. Importantly, they are inherently brittle and undergo catastrophic failure modes. This often results in designs where high safety margins are employed (and thus added weight). In addition, traditional long fibre reinforced composite materials have time consuming manufacturing processes and poor recyclability which has limited their use in automotive applications.

Various efforts have been made in order to develop recyclable and ductile composite materials [1-3]. One approach is to make both the fibres and the matrix using a single recyclable ductile polymer. This emerging group of composites are termed self-reinforced polymer composites (or single-polymer composites). Although these composites have excellent recyclability and ductility, they have a lower stiffness and strength compared to traditional glass or carbon fibre

\footnotetext{
$1 *$ Corresponding author, Tel.: +46 (0) 734612498

Email address: schnei@kth.se
} 
composites [4]. However, their lower density (typically between $800-1400 \mathrm{kgm}^{-3}$ ) compared to traditional composites implies that these self-reinforced polymer composites are still competitive on a specific strength or stiffness basis.

To-date, research in the field of self-reinforced polymer composites $(\mathrm{SrP})$ has focused on developing manufacturing processes for laminates and investigating the quasi-static properties of the material [2,5]. There is limited data on utilising the recyclability of SrPs to create fully recyclable structures or on utilising the high ductility of the $\mathrm{SrPs}$ in impact protection applications via the use of cellular structures [6,7]. The aim of the present paper is to fill these gaps in the literature. The outline of the paper is as follows. We shall first describe a manufacturing route to construct a fully recyclable corrugated sandwich structure. Second, we will present the dynamic loading response of both the SrP material and the corrugated sandwich core. Finally, we will compare and contrast the response of the SrP cellular core with competing polymeric foam cores.

\section{Materials and manufacturing route}

\subsection{Description of constituent material}

The material used in this study is a self-reinforced poly(ethylene terephthalate) composite (SrPET). It comprises of commingled yarns with 50 weight\% amorphous PET fibres (which are chemically modified to melt at $160-180^{\circ} \mathrm{C}$ and will be used as the matrix material) and 50 weight\% high tenacity PET reinforcement (which will serve as the reinforcing fibres with a melting temperature of $260^{\circ} \mathrm{C}$ ). The yarns are woven to form a balanced $2 / 2$ twill fabric with an areal weight of $0.710 \mathrm{kgm}^{-2}$ (supplied by Comfil@ ApS [8]). Both fibre and matrix have a density of $1380 \mathrm{kgm}^{-3}$.

\subsection{Manufacture of the flat laminates}

Prior to consolidating the twill fabric into a laminate, the PET fabric needs to undergo a dehumidification process as moisture in the PET material leads to increased brittleness. The fabric was dried by placing it in a climate chamber for $24 \mathrm{~h}$ at relative humidity of $15 \%$ and a temperature of $50^{\circ} \mathrm{C}$. Immediately after the dehumidification process, the fabrics were stacked in the thickness direction and consolidated in a hot-press under 1 bar pressure above the ambient pressure. Heating was applied at a rate of $10^{\circ} \mathrm{C} / \mathrm{min}$ up to $220^{\circ} \mathrm{C}$ after which the temperature was held constant for 20 minutes and subsequently cooled at $10^{\circ} \mathrm{C} / \mathrm{min}$ back to room temperature. This process produced a laminate in which each twill fabric layer was $0.5 \mathrm{~mm}$ thick. 


\subsection{Manufacture of the corrugated sandwich panels}

Corrugated sandwich panels of SrPET were manufactured in three steps. First, pre-dried layers of SrPET fabric were stacked in an aluminium mould to form the bottom face sheet as shown in (1) in Fig. 1. Next aluminium moulds with an isosceles trapezoidal cross-section of height of $h=20 \mathrm{~mm}$, base length of $50 \mathrm{~mm}$ and an inclination angle of $\omega=45^{\circ}$ (see inset of Fig. 1) were manufactured and these profiles were coated with a Tygovac RF260 Flouropolymer FEP release film in order to ensure successful de-moulding. The pre-dried SrPET fabric was then wrapped around these moulds so that the weft yarns of the fabric were bent over the profile edges and the warp yarns remained straight (see (2) in Fig. 1). The start and the end of the fabric wrappings were always placed in the middle of the base (longer parallel edge) of the trapezoid. Once all aluminium profiles had been wrapped with fabric, they were placed on top of the lower face sheet fabric in an alternating manner so as to form a space-filling arrangement. Finally the layers for the top face sheet were stacked on top of the core (see (3) in Fig. 1).

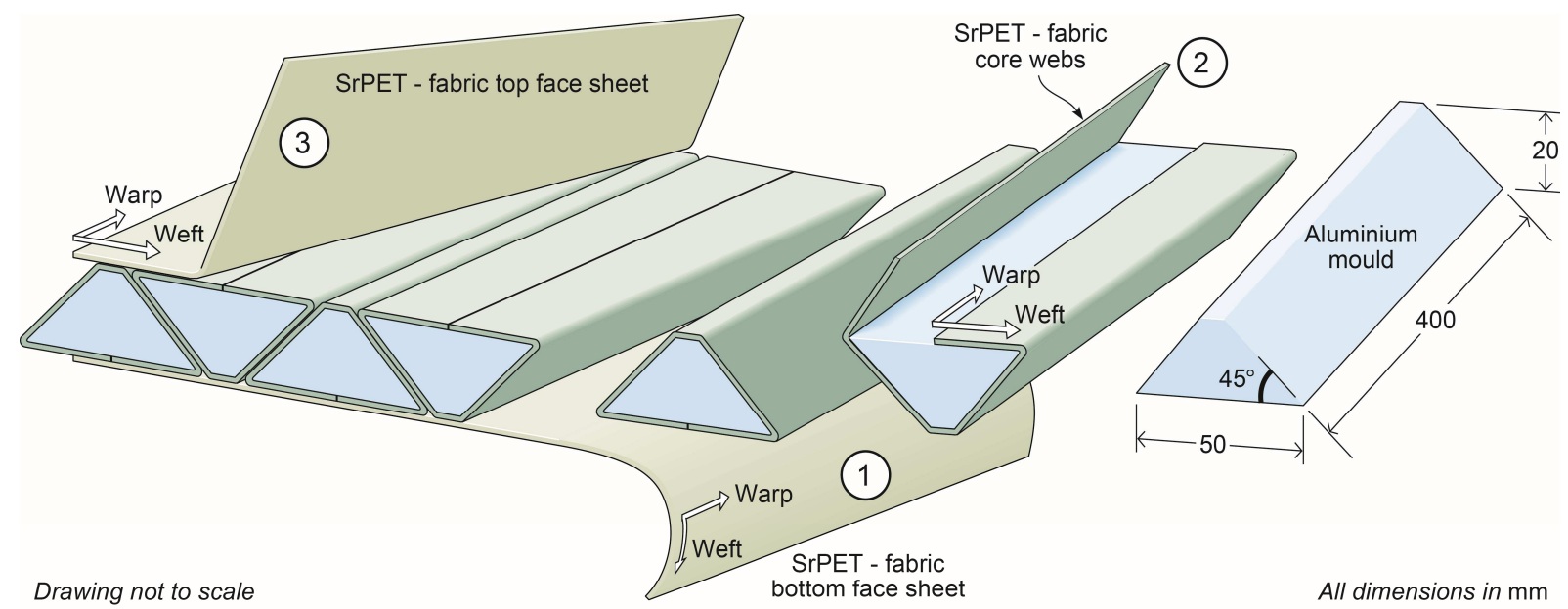

Figure 1: Schematic illustration of three of the assembly steps used to manufacture the SrPET corrugated sandwich panels. (1) placement of the bottom face sheet fabrics. (2) Wrapping of fabric around the trapezoidal aluminium moulds and placement of the moulds into the tool to form the core webs. (3) Placement of the top face sheet fabrics.

The stack of wrapped profiles and face sheets was then placed in a hot-press where consolidation was performed following the same pressure and heating procedure described in section 2.2 for the flat laminate. After the consolidation the aluminium profiles were demoulded to obtain a sandwich plates as shown in Fig. 2a. The various dimensions of the corrugated core are labelled in Fig. $2 \mathrm{~b}$ and the dimensions of the three corrugated core geometries manufactured and tested in this study listed in Table 1. Several optical micrographs 
were taken to check the consolidation process of the corrugated core but little/no porosity was detected. An optical micrograph of the joint between the corrugated core and the face-sheet is included in Fig. 2c to illustrate the alignments of the fibre tows in the joint.

\subsection{Specimens for material property testing}

The compressive response of the SrPET material was measured using cubical specimens of side $16 \mathrm{~mm}$ over applied strain rates ranging from $10^{-4} \mathrm{~s}^{-1}$ to $10^{3} \mathrm{~s}^{-1}$. The specimens were cut-out from a $16 \mathrm{~mm}$ thick flat laminate and the loading surfaces of the specimens were machined flat and parallel to ensure a proper load introduction. In all cases, compressive loading was measured in the weft fibre direction.
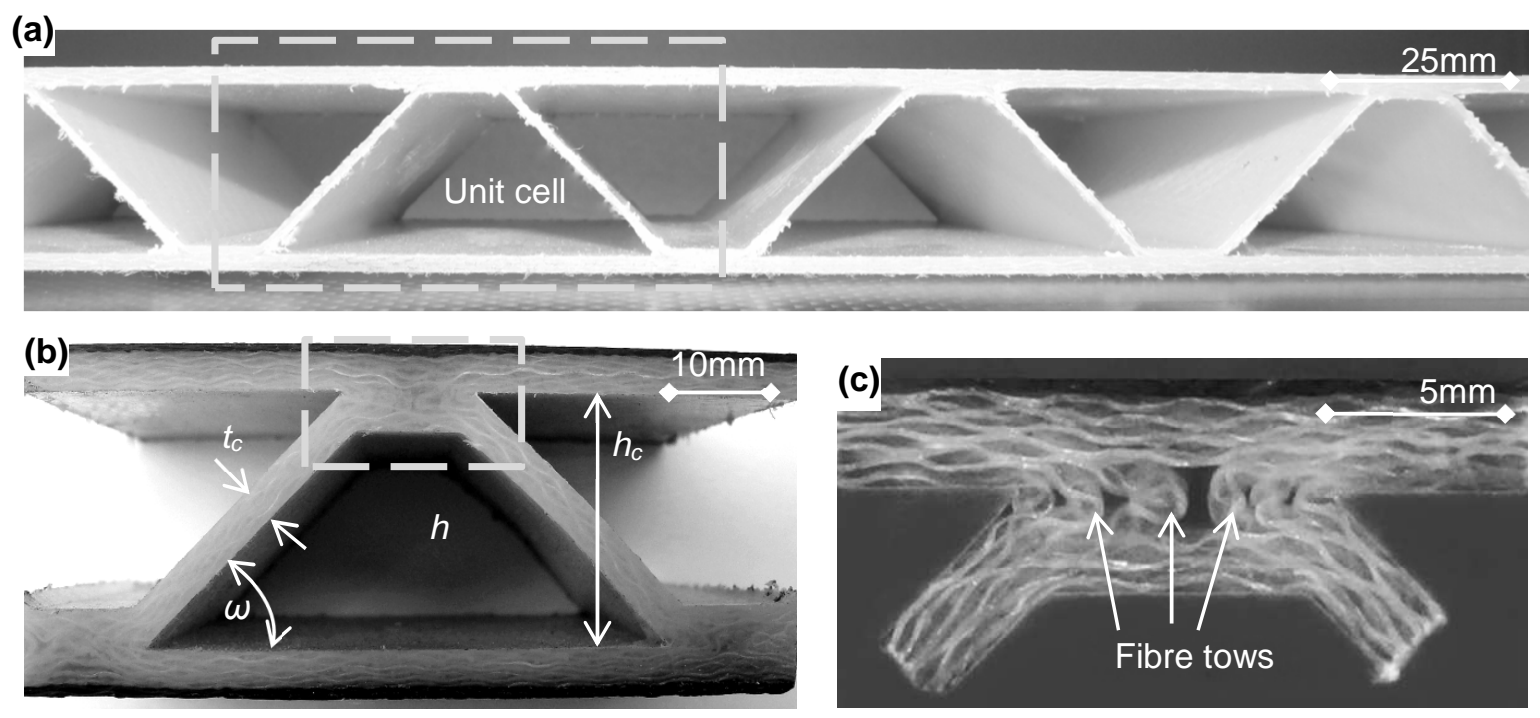

Figure 2: Optical photographs of (a) the as manufactured SrPET corrugated sandwich plate, (b) a unit-cell of the corrugated structure and (c) a close-up view of the core web and face sheet joint where the fibres from the core are folded into the face sheet showing resin rich joints.

\subsection{Corrugated specimens for structural property testing}

Three corrugated core geometries were investigated in this study. The focus here is on the compressive properties of the corrugated core and hence, the sandwich face sheets were reinforced with one layer of a carbon fibre fabric on the outer sides so as to ensure minimal deformation of the face sheets during compression of the core. The sandwich panels were cut into unit cell specimens comprising a single corrugation (Fig. 2a); and Table 1 summarises the geometrical dimensions of the three configurations investigated here. All three geometries had unit cells of length $L \approx 62 \mathrm{~mm}$ and mould height $h=20 \mathrm{~mm}$. The strut thickness $t_{c}$ of the struts was varied so as to change density of the corrugated core. The smeared-out density, $\rho_{c}$, of the corrugated core in terms of the parameters defined in Fig. $2 \mathrm{c}$ is given as 


$$
\rho_{c}=\frac{\frac{h t_{c} 2}{\sin (\omega)}+\left(h_{c}-h\right)(L-2 h)}{L h_{c}} \rho_{m},
$$

where $h$ is the height of the aluminium mould used during the consolidation process, $t_{c}$ is the core member thickness, $\omega$ is the corrugation angle, $h_{c}$ is the resulting sandwich core height, $L$ is the unit cell length and $\rho_{m}$ is the density of the consolidated SrPET solid material. The three corrugated cores have densities $\rho_{c}$ in the range $78 \mathrm{kgm}^{-3}, 150 \mathrm{kgm}^{-3} 241 \mathrm{kgm}^{-3}$. We define the aspect ratio of the core struts to be $t_{c} / \ell$, where $\ell=h_{c} / \sin \omega$ is the length of the core strut, and subsequently referred to the cores via the aspect ratio of the core struts as slender, intermediate and stubby as listed in Table 1.

\begin{tabular}{lccccccc}
\hline $\begin{array}{l}\text { Core } \\
\text { Type }\end{array}$ & $\begin{array}{c}t_{c} \\
(\mathrm{~mm})\end{array}$ & $t_{c} / \ell$ & $\begin{array}{c}h_{c} \\
(\mathrm{~mm})\end{array}$ & $\begin{array}{r}L \\
(\mathrm{~mm})\end{array}$ & $\begin{array}{r}\omega \\
\left({ }^{\circ}\right)\end{array}$ & $\begin{array}{r}h \\
(\mathrm{~mm})\end{array}$ & $\begin{array}{c}\rho_{c} \\
\left(\mathrm{~kg} \mathrm{~m}^{-3}\right)\end{array}$ \\
\hline Slender & 1.0 & 0.04 & 20.8 & 61.6 & 45 & 20 & 78 \\
\hline Intermediate & 2.1 & 0.07 & 21.3 & 63.1 & 45 & 20 & 150 \\
\hline Stubby & 3.1 & 0.11 & 24.1 & 64.7 & 45 & 20 & 241 \\
\hline
\end{tabular}

Table 1: Geometry of the corrugated unit cells

\section{Experimental protocol}

To accommodate the large range of loading rates investigated here four different test methods were employed. At low loading rates (applied compressive velocities $v_{0}<0.005 \mathrm{~ms}^{-1}$ ), a traditional Instron 4045 screw-driven test machine was used. At intermediate loading rates $\left(0.005 \mathrm{~ms}^{-1}<v_{0}<1 \mathrm{~ms}^{-1}\right)$, a servo-hydraulic Schenk PSA machine was used and at high loading rates $\left(v_{0}>1 \mathrm{~ms}^{-1}\right)$ both an Instron VHS 160/100-20 and a Kolsky bar setup were used. Each specimen configuration was tested at least three times for each loading velocity and the average data is reported. For experiments conducted using the Kolsky bar, one specimen per loading velocity and configuration was tested.

\subsection{Quasi - static loading rates}

A screw-driven uniaxial loading machine (Instron 4045) equipped with a $100 \mathrm{kN}$ or a $30 \mathrm{kN}$ load cell was used depending on the expected failure load. A Redlake Motion ProX ${ }^{\mathrm{TM}}$ high speed camera system was used to capture 100 images per second while simultaneously recording the corresponding force from the load cell. The image sequence was used to infer the surface strains by using the commercial digital image correlation (DIC) software Aramis GOM [9]. This test 
procedure was used to perform compression tests on the SrPET solid material and corrugated core for strain rates in the range $\dot{\varepsilon} \leq 0.5 \mathrm{~s}^{-1}$ and $\dot{\varepsilon} \leq 0.2 \mathrm{~s}^{-1}$, respectively.

\subsection{Intermediate loading rates}

For intermediate loading rates, a servo-hydraulic uniaxial testing machine Hydropuls PSA from Schenck equipped with a $40 \mathrm{kN}$ load cell was used. The applied strain was measured using the same equipment as for the quasi-static experiments but images were captured at a frame rate of 1000 images per second. This test procedure was used to perform compression tests on the SrPET solid material and corrugated core for strain rates in the range $0.5 \mathrm{~s}^{-1} \leq \dot{\varepsilon} \leq 100 \mathrm{~s}^{-1}$ and $0.2 \mathrm{~s}^{-1} \leq \dot{\varepsilon} \leq 40 \mathrm{~s}^{-1}$, respectively.

\subsection{High loading rates}

To apply strain rates $\dot{\varepsilon} \geq 100 \mathrm{~s}^{-1}$ and $\dot{\varepsilon} \geq 40 \mathrm{~s}^{-1}$ on the SrPET solid material and corrugated core specimens, two different test setups were employed. The reproducibility of the measurements between these two setups helped establish confidence in these high loading rate measurements.

High speed servo-hydraulic test machine

A servo-hydraulic Instron VHS 160/100-20 with a maximum load capacity of $20 \mathrm{kN}$ was used to compress specimens at velocities $v_{0}$ in the range $0.1-20 \mathrm{~ms}^{-1}$. The load was measured using a piezoelectric load cell while the imposed nominal strain rate was given as $v_{0} / H$ where $H$ is the specimen height. The nominal strain then follows as $v_{0} t / H$ where time $t=0$ corresponds to the instant the loading platen impacts the specimen. High speed photography, using a Phantom v.1600.1 camera, was to confirm the accuracy of the above definitions of strain rate and strain.

\section{Direct impact Kolsky bar tests}

A gas gun setup was used to fire projectiles at velocities between $10-50 \mathrm{~ms}^{-1}$ that impacted the specimens placed on a Kolsky bar. The load transmitted through the specimen into the Kolsky bar was measured via strain gauges and used to infer the dynamic compressive stress within the specimen. Again, high speed photography via a Phantom v.1600.1 camera was used to measure the applied strains. A more detailed description of the Kolsky bar setup for both material and corrugated core testing is given in [10].

In high rate loading tests, axial wave effects imply that axial equilibrium front (surface being displaced at velocity $v_{0}$ ) and stationary rear surface may not be attained. However, in all the measurements reported here the loading rates are sufficiently low that specimens axially equilibrated early in the loading history. This was confirmed by (i) measurements of the load 
on the front and rear surfaces of the specimens in the Instron VHS 160/100-20 tests and (ii) by performing "front" and "rear" impact tests using the direct impact Kolsky bar; see Kazemahvazi et al. [10] for further details. Examples of load-displacement histories confirming the axial equilibrium are presented in Fig. 3 through comparisons between the measurements made for the "front" and "rear" impact test. For the forthcoming graphs we only present single stress versus strain curves for all the loading rates investigated here to enhance graphical clarity. Given that wave effects are negligible in the tests reported, we argue that the responses reported here are representative of material behaviour.

\subsection{Validation of test method accuracy}

In order to establish the fidelity of the measurements, the Instron VHS 160/100-20 was used to perform a limited number of additional tests at compression velocities in the range of the Kolsky bar setup (10-20ms $\left.{ }^{-1}\right)$. These Instron VHS 160/100-20 tests gave results nearly identical (to within the scatter inherent in the SrPET parent material) to the original Kolsky bar tests, see examples of comparisons of load-displacement histories for the two different high rate test methods in Fig. 3.

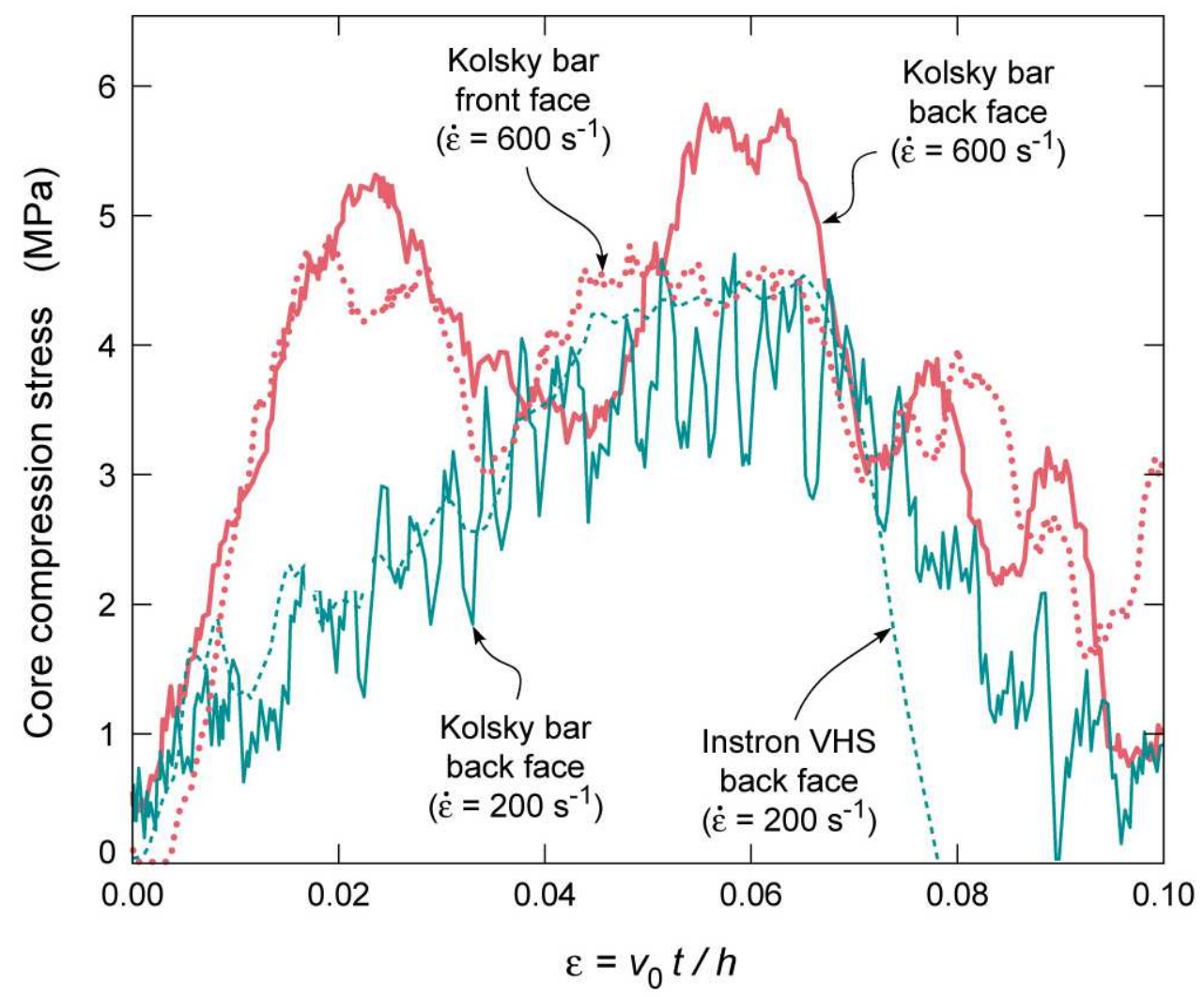

Figure 3: The nominal compressive stress versus strain response of the intermediate corrugated core configuration showing front face and back face response. In addition the response from different testing setups (Kolsky bar and Instron VHS) at the same compression strain rate is presented. 


\section{Dynamic compressive response of the SrPET material}

The measured uniaxial true compressive stress versus logarithmic strain curves at an applied strain rate between $\dot{\varepsilon}=10^{-4}-10^{3} \mathrm{~s}^{-1}$ are plotted in Fig. 4. For completeness, the uniaxial true tensile stress versus logarithmic strain at an applied strain rate of $\dot{\varepsilon}=10^{-4} \mathrm{~s}^{-1}$ is also included in Fig. 4a [4]. We observe three phases of deformation for all compression loading rates. In the first phase there is an elastic response followed by a plastic hardening phase up to a point of maximum load. In the final phase, the stress decreases due to significant macroscopic material damage.

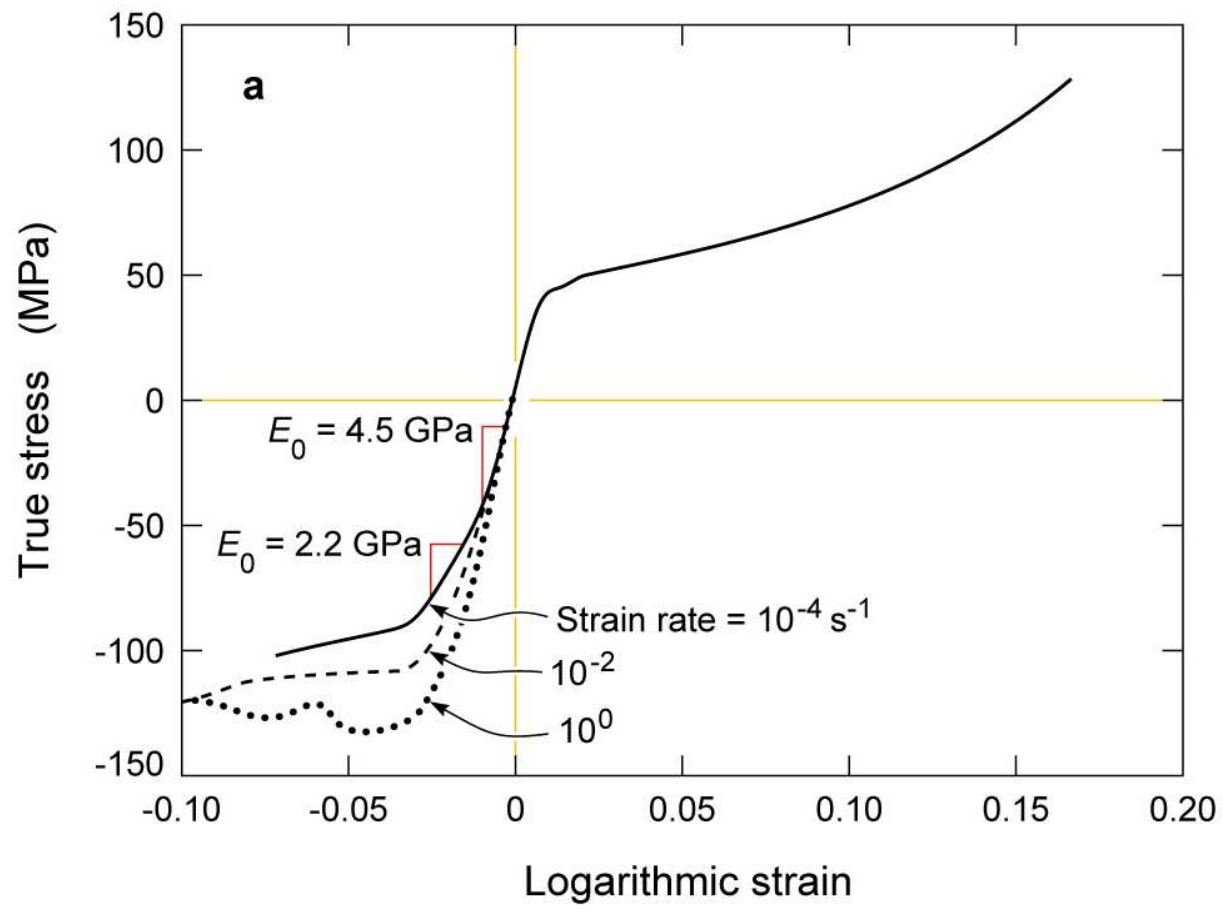




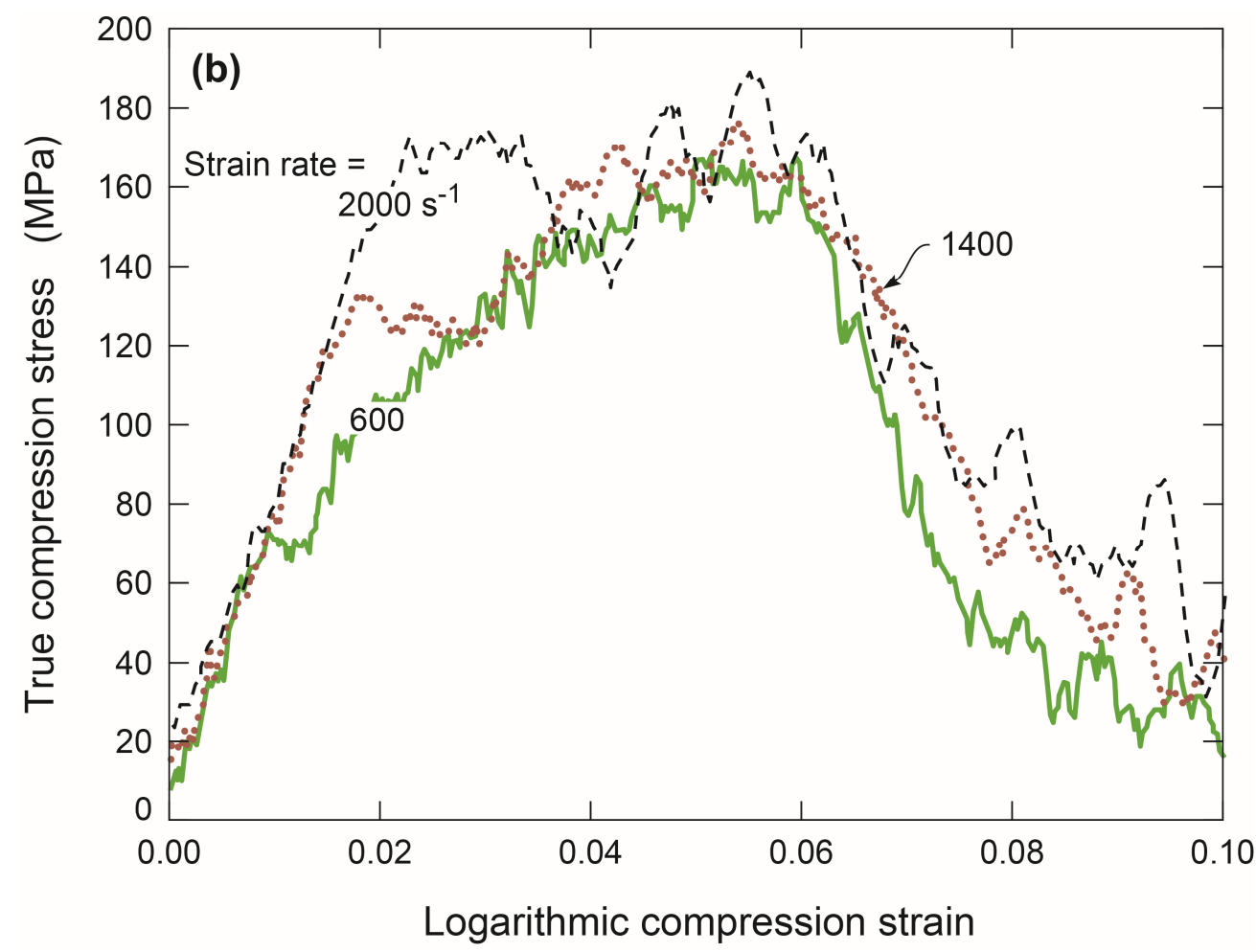

Figure 4: The measured uniaxial compressive response of the solid SrPET material at (a) low and intermediate strain rates (including the tensile response at the lowest tested strain rate [4]) and (b) high strain rates.

\subsection{Elastic phase - change of stiffness with strain rate}

The change in stiffness as function of strain rate is presented in Fig. 5 as the dynamic stiffness enhancement ratio $E_{D} \equiv E(\dot{\varepsilon}) / E_{0}$, where $E_{0}$ is the quasi-static loading stiffness $\left(E_{0}=\right.$ $4.5 \mathrm{GPa}$ ) and $E$ is the dynamic loading stiffness at an applied strain rate $\dot{\varepsilon}$. We observe an approximately linear increase in stiffness (on a logarithmic $x$-axis) up to $E=9 \mathrm{GPa}$ at an applied strain rate of $\dot{\varepsilon}=10^{3} s^{-1}$ (i.e. $E_{D}=2$ ). This level of stiffness enhancement is similar to the observations made in previous studies for other polymeric materials $[11,12]$ where a stiffness enhancement factor of two was found for polycarbonate (PC), poly(methyl methacrylate) (PMMA) and polyamideimide (PAI) over the strain rate range of $10^{-3}$ $10^{3} s^{-1}$ 


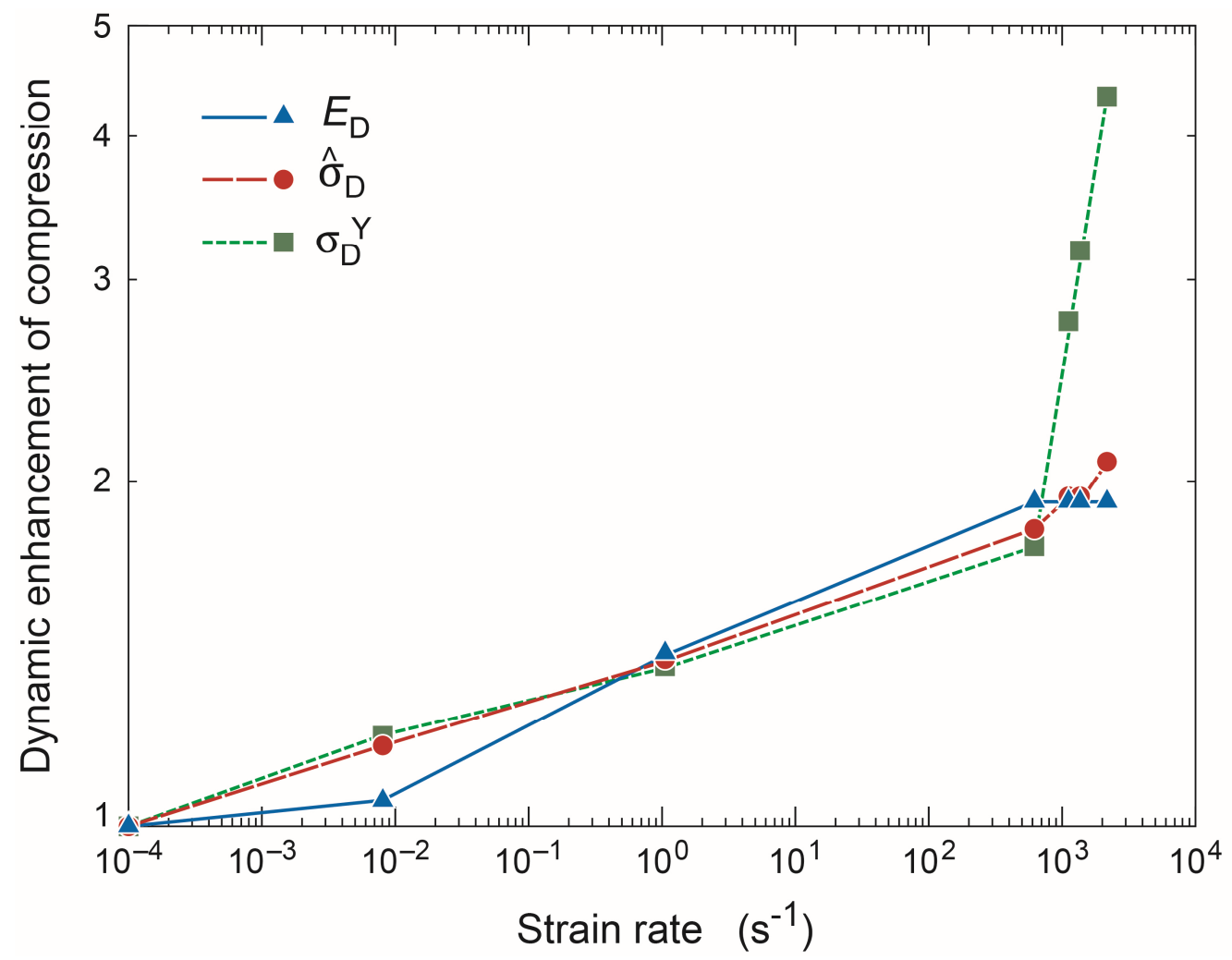

Figure 5: The dynamic compression enhancement ratios $E_{D}=E\left(\varepsilon^{\cdot}\right) / E_{0}, \hat{\sigma}_{D}=\hat{\sigma}(\dot{\varepsilon}) / \hat{\sigma}_{0}$ and $\sigma_{D}^{Y}=\sigma^{Y}(\dot{\varepsilon}) / \sigma_{0}^{Y}$ for the modulus, yield strength and peak strength respectively of the solid SrPET material as a function of the compressive strain rate $\dot{\varepsilon}$.

\subsection{Yield phase - change of yield point with strain rate}

The yield strength $\sigma^{Y}$ is taken as the $0.2 \%$ offset strength and then we define a dynamic yield enhancement as $\sigma_{D}^{Y} \equiv \sigma^{Y}(\dot{\varepsilon}) / \sigma_{0}^{Y}$, where $\sigma_{0}^{Y}$ is the quasi-static yield strength $\left(\sigma_{0}^{Y}=44 \mathrm{MPa}\right)$ and $\sigma^{Y}$ is the dynamic loading yield strength at an applied strain rate $\dot{\varepsilon}$. This strength enhancement ratio is plotted in Fig. 5 and shows that $\sigma_{D}^{Y}$ increases linearly (on a logarithmic $x$ axis) with $\dot{\varepsilon}$ up to a transition strain rate of $\dot{\varepsilon}_{T}=10^{2} \mathrm{~s}^{-1}$ beyond which there is a dramatic increase in $\sigma_{D}^{Y}$. This sudden increase of the dynamic yield strength is related to the decrease of molecular mobility of the polymer chains. At a certain combination of material temperature and applied loading rate, the polymer chains "lock up" and this results in significant increase of yield strength. This effect is seen for a range of polymeric materials. For example, PC and PAI polymers exhibit similar behaviours and at room temperature; the transition strain rates for PC and PAI is $\dot{\varepsilon}_{T} \approx 10^{2} s^{-1}[11,12]$ similar to the PET investigated here.

Photographic sequences for the deformation of the SrPET solid specimens at a range of applied strain rates are included in Fig. 6. We emphasize here that regardless of the applied loading rate, no macroscopic damage is observed at the onset of yield. 


\section{Un-loaded Yield point $\quad$ Peak point $\quad$ Post peak}

point

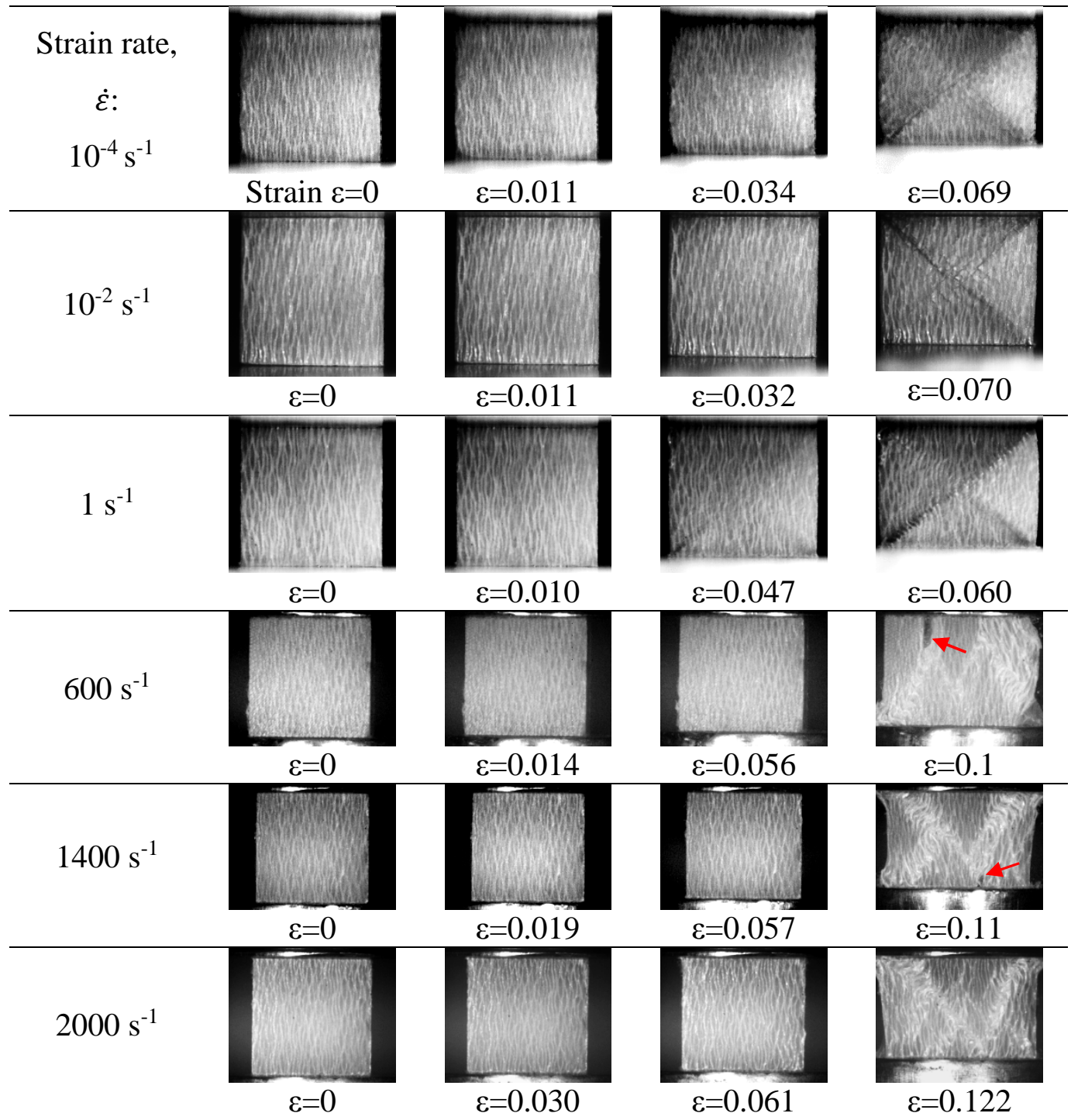

Figure 6: Montage of photographs showing the compressive deformation of the solid SrPET material at three levels of compressive strain $\varepsilon$ for selected values of the applied strain rate $\dot{\varepsilon}$ ranging from quasi-static to $\dot{\varepsilon}=2000 \mathrm{~s}^{-1}$.

\subsection{Effect of strain rate of the peak strength and failure mode}

The stress versus strain histories plotted in Fig. 4 show that for strain rates $\dot{\varepsilon}>10^{2} \mathrm{~s}^{-1}$ there is a large increase in the material strength while, no distinct peak load is observed at the lower strain rates with the material displaying a strength plateau. The differences in the deformation modes in low and high loading rates are also apparent in Fig. 6 . At loading rates $\dot{\varepsilon} \leq 1 \mathrm{~s}^{-1}$, two $45^{\circ}$ shear bands form with deformation within these bands occurring by the plastic shearing of the matrix as well as plastic bending of the fibres. On the other hand, at high loading rates $\dot{\varepsilon}>$ 
$10^{2} \mathrm{~s}^{-1}$, catastrophic failure of the specimen due to delamination is observed (indicate by red arrows in Fig. 6). We rationalise this by recalling that the yield strength of the material is strongly rate sensitive at strain rates in excess of $10^{2} \mathrm{~s}^{-1}$. This increases the resistance of the fibres to plastic bending and inhibits the formation of shear bands: we surmise that this suppressing of the benign shear band mode induces the catastrophic delamination mode.

Similar to the modulus and yield strength we define a dynamic peak strength enhancement as $\hat{\sigma}_{D} \equiv \hat{\sigma}(\dot{\varepsilon}) / \hat{\sigma}_{0}$, where $\hat{\sigma}_{0}$ is the quasi-static peak strength $\left(\hat{\sigma}_{0}=90 \mathrm{MPa}\right)$ and $\hat{\sigma}$ the dynamic peak strength at an applied strain rate $\dot{\varepsilon}$. This peak strength enhancement is included in Fig. 5 . In line with the modulus enhancement, $\hat{\sigma}_{D}$ increases linearly with $\log (\dot{\varepsilon})$ with $\hat{\sigma}_{D}$ attaining a maximum value of 2 over the range of strain rates investigated here.

\section{Dynamic compressive response of the corrugated sandwich cores}

We now proceed to present measurements of the compressive response of the corrugated core for nominal strain rates in the range $10^{-4} \mathrm{~s}^{-1}$ to $10^{3} \mathrm{~s}^{-1}$. We first present a discussion on the quasi-static collapse modes of the corrugated core and then proceed to discuss the change in these modes with imposed strain rates for the three core densities investigated here.

\subsection{Quasi-static collapse modes and strength}

There are three operative collapse modes for corrugated cores subjected to uniaxial compression as justified by Cote et al. [13]: (i) Elastic buckling of the core struts; (ii) plastic buckling of the core struts or (iii) collapse of the core struts at the solid material ultimate strength $\hat{\sigma}_{0}$. Simple analytical expressions are now given for each of these modes under quasi-static loading conditions.

Define the compressive stress on the corrugated core as $\sigma \equiv P / A$, where $P$ is the applied outof-plane compressive force on the single unit cell of the corrugated core (Fig. 2b) and $A=L b$, where $b$ is the width of the unit cell in the warp direction. For $t_{c} / \ell \ll 1$, the contribution to the core strength from the bending of the struts is negligible and the simple force balance dictates that the applied stress $\sigma$ is related from the axial stress $\sigma_{c m}$ in the core struts via the relation

$$
\sigma=2\left(\frac{t_{C}}{L}\right) \sigma_{c m} \sin \omega
$$

The quasi-static collapse strength $\sigma_{p}$ of the corrugated core is obtained by setting $\sigma_{c m}=\hat{\sigma}_{c m}$, where

$$
\hat{\sigma}_{c m}=\min \left(\hat{\sigma}_{0}, \hat{\sigma}_{b}\right) \text {, }
$$


with $\hat{\sigma}_{b}$ being the buckling stress of the core strut. Buckling failure of the core strut of length $\ell$ can occur in either the elastic or plastic regime. This is specified in terms of the Young's modulus $E$ and plastic tangent modulus $E_{t}$ of the solid SrPET materials as

$$
\hat{\sigma}_{b}=\left\{\begin{array}{lc}
\frac{E \pi^{2} n^{2}}{3}\left(\frac{t_{c}}{\ell}\right)^{2} & \text { if } \frac{t_{c}}{\ell}<\sqrt{\frac{3 \sigma_{0}^{Y}}{E \pi^{2}}} \\
\frac{E_{t} \pi^{2} n^{2}}{3}\left(\frac{t_{c}}{\ell}\right)^{2} & \text { otherwise. }
\end{array}\right.
$$

In the above formulae, where $n$ defines the buckling mode shape, we have assumed that the boundary conditions of the struts are built-in (clamped) and that the plastic tangent modulus (i.e. the slope of the uniaxial quasi-static true stress versus logarithmic strain curve beyond the yield strength $\sigma_{0}^{Y}$ ) is a constant. This is consistent with the data in Fig. 4 which suggests that $E_{t} \approx 2.2 \mathrm{GPa}$.

The above relations specify the collapse strength of the corrugated core under quasi-static loading conditions. Recall that shock/wave effects play a negligible role for the loading rates employed here with the specimens always in axial equilibrium. Thus, three main effects are expected to enhance the core strength under high rates of loading: (i) increase in the modulus of the solid SrPET material with strain rate; (ii) increase in the SrPET material yield strength with strain rate and (iii) micro-inertial stabilisation of the struts of the corrugated core against buckling [14]. The effects (i) and (ii) are material effects and independent of the corrugated core geometry while (iii) will depend strongly on the corrugated core strut aspect ratio $t_{c} / \ell$. We thus proceed to discuss the compressive response of the three corrugated core geometries in turn in order of increasing strut aspect ratio. Results are presented in terms of the applied stress $\sigma$ versus strain $\varepsilon \equiv v_{o} t / h_{c}$, where time $t=0$ corresponds to the instant at which we begin to

displace the face sheets of the corrugated core at a velocity $v_{o}$. The applied strain rate is $\dot{\varepsilon} \equiv$ $v_{o} / h_{c}$.

\subsection{Dynamic out-of-plane compressive response of the slender core configuration}

Under quasi-static loading conditions $\left(10^{-4} \mathrm{~s}^{-1} \leq \dot{\varepsilon} \leq 10^{-2} \mathrm{~s}^{-1}\right)$ the $\sigma$ versus strain $\varepsilon$ displays an initial elastic regime followed by a stress plateau at $\sigma \approx 0.32 \mathrm{MPa}$ as seen in Fig. 7. The deformation mode is included in Fig. 8 via a series of images at different stages in the deformation. It is clear that collapse occurs by the buckling of the struts in the $1^{\text {st }}$ Euler mode ( $n=1$ in eq.(4) ). Consistent with this observation the analysis of Section 5.1 predicts $\sigma_{p}=$ $0.45 \mathrm{MPa}$ with the operative collapse mode being elastic buckling. We rationalise the slightly 
lower observed collapse stress to imperfections in the as-manufactured corrugated core struts that are not accounted for in the analysis of Section 5.1.

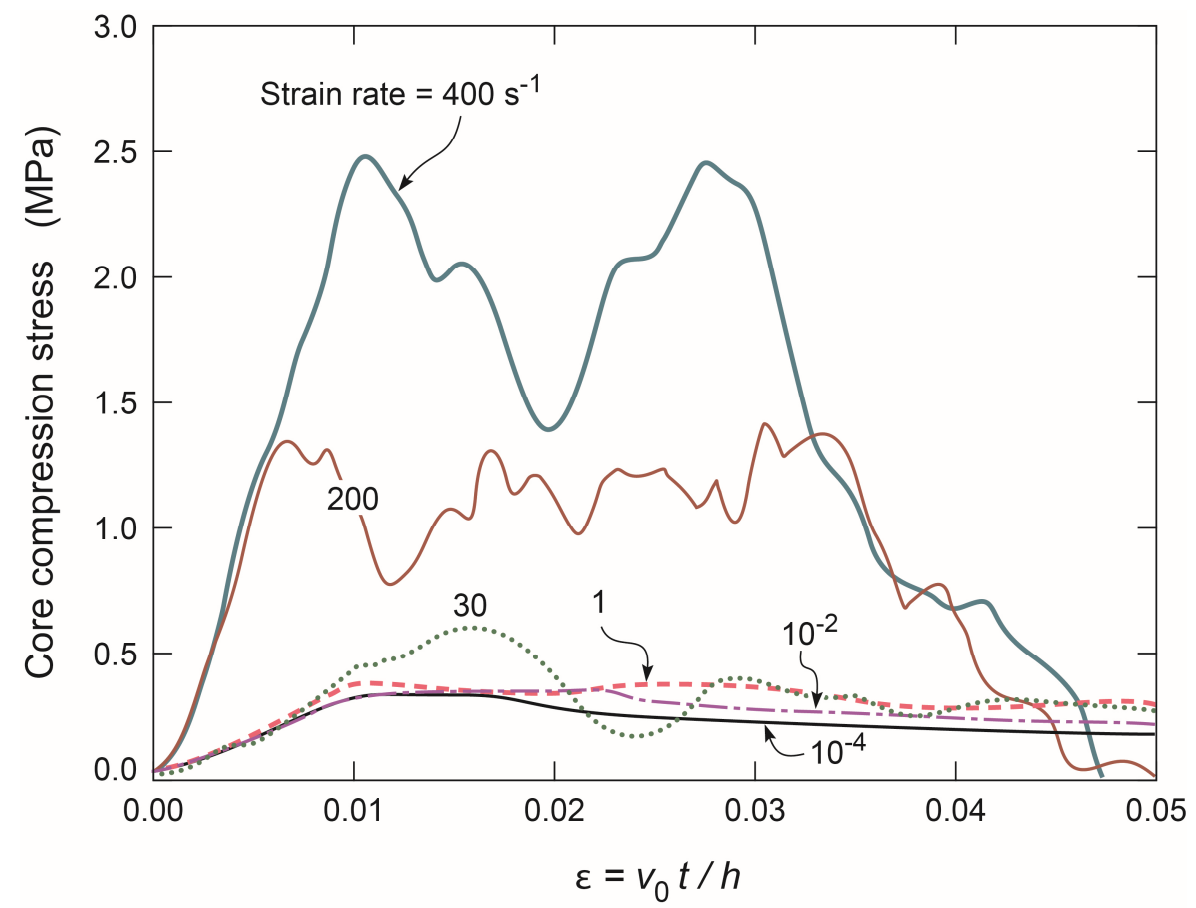

Figure 7: The nominal compressive stress versus strain response of the slender corrugated core configuration.

No significant change in the $\sigma$ versus strain $\varepsilon$ response is observed for strain rates up to $\dot{\varepsilon}=$ $1 \mathrm{~s}^{-1}$. However, at $\dot{\varepsilon}=200 \mathrm{~s}^{-1}$ both the initial stiffness and collapse strength of the corrugated core is substantially higher compared to quasi-static loading conditions. With a further increase in $\dot{\varepsilon}$ to $400 \mathrm{~s}^{-1}$ there is no increase in the stiffness but the collapse strength is seen to rise further. Recall that over the range of strain rates investigated here, the solid SrPET material modulus $E$ and yield strength $\sigma_{0}^{Y}$ increase by a factor of 2 and 4.5, respectively. The observed stiffness increase with increasing $\dot{\varepsilon}$ is therefore due to the increase in the modulus of the solid material with increasing strain rate. However, Eq. (4) dictates that the slender corrugated core will collapse by elastic buckling even with material strain rate effects accounted for. This implies that the collapse strength of the corrugated core should increase by no more than a factor of two from quasi-static loading conditions to the highest loading rate shown in Fig. 8. The experimental data suggests that $\sigma_{p}$ increases by a factor of 5 as $\dot{\varepsilon}$ is increased from $10^{-4} \mathrm{~s}^{-1}$ to $400 \mathrm{~s}^{-1}$. To rationalise this observation the examined collapse modes of the corrugated core is shown in Fig. 8. 


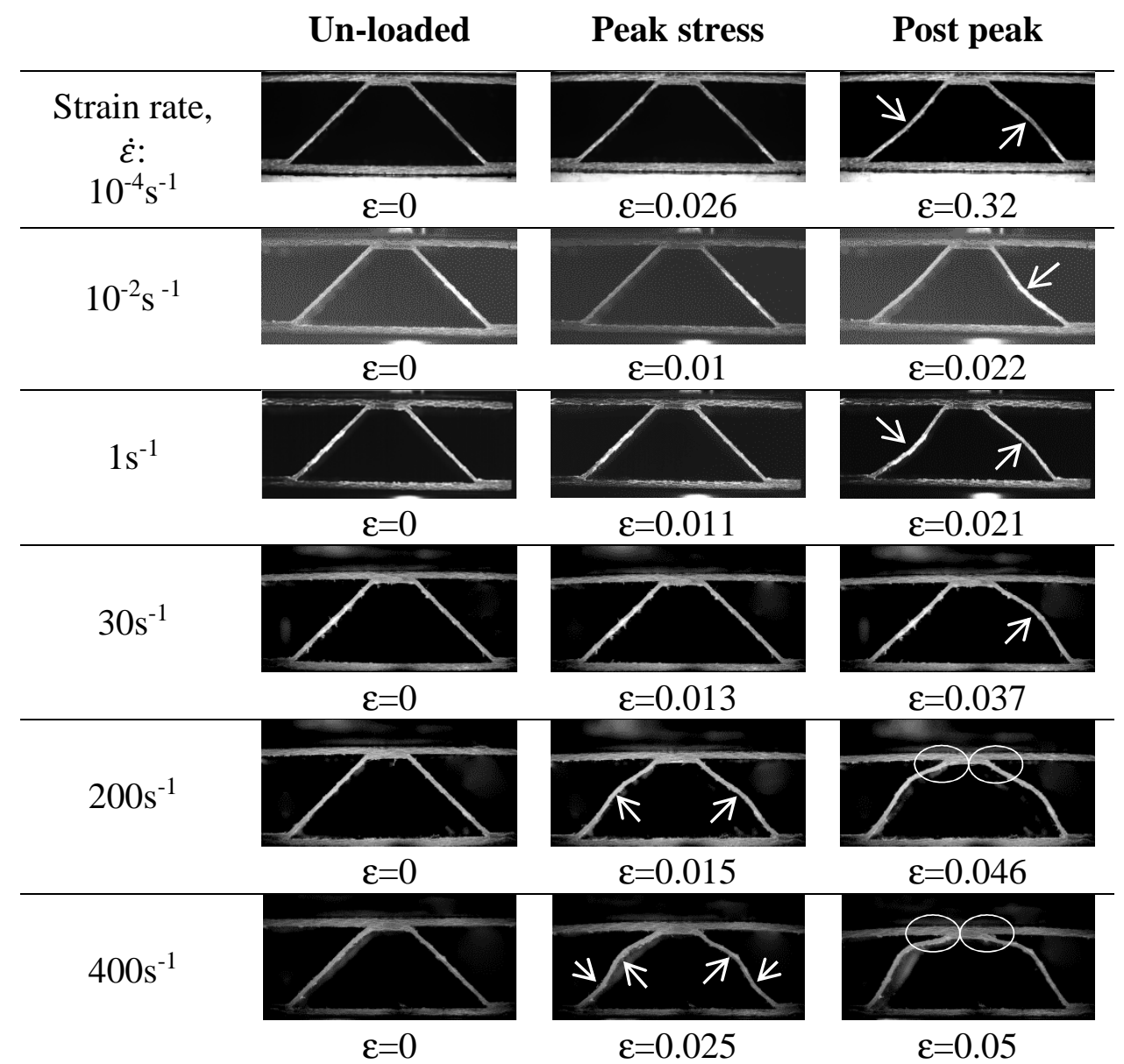

Figure 8: Montage of photographs showing the compressive deformation of the slender corrugated core configuration at three levels of compressive strain $\varepsilon$ for selected values of the applied strain rate $\dot{\varepsilon}$ ranging from quasi-static to $\dot{\varepsilon}=400 \mathrm{~s}^{-1}$.

While it is clear that under quasi-static conditions collapse was by the buckling of the core struts in the $1^{\text {st }}$ Euler mode, higher order collapse modes (corresponds to $n=2$ or $n=3$ in eq.(4)) are evident from the high-speed photographs of the specimens deformed at $\dot{\varepsilon}=200 \mathrm{~s}^{-1}$ and $400 \mathrm{~s}^{-1}$ (see arrows in Fig 8). This change in the buckling mode occurs due to micro-inertial stabilisation of the struts against buckling as discussed in $[10,14]$ and observed in experimental and numerical investigations of metallic corrugated cores [15]. It is this micro-inertial stabilisation that causes the large observed increase in the collapse strength of the SrPET corrugated core with slender struts.

\subsection{Dynamic out-of-plane compressive response of intermediate core configuration}

Under quasi-static loading conditions $\left(10^{-4} \mathrm{~s}^{-1} \leq \dot{\varepsilon} \leq 10^{-2} \mathrm{~s}^{-1}\right)$ the $\sigma$ versus $\varepsilon$ response (Fig. 9) shows an initial linear elastic response followed by a distinct stress peak at about 2.1 MPa as seen in Fig. 9. The analysis of Section 5.1 suggests that at this intermediate slenderness ratio, 
collapse will occur by plastic buckling at $1.9 \mathrm{MPa}$ in good agreement with the measured value and the collapse mode seen in the photographs in Fig. 10.

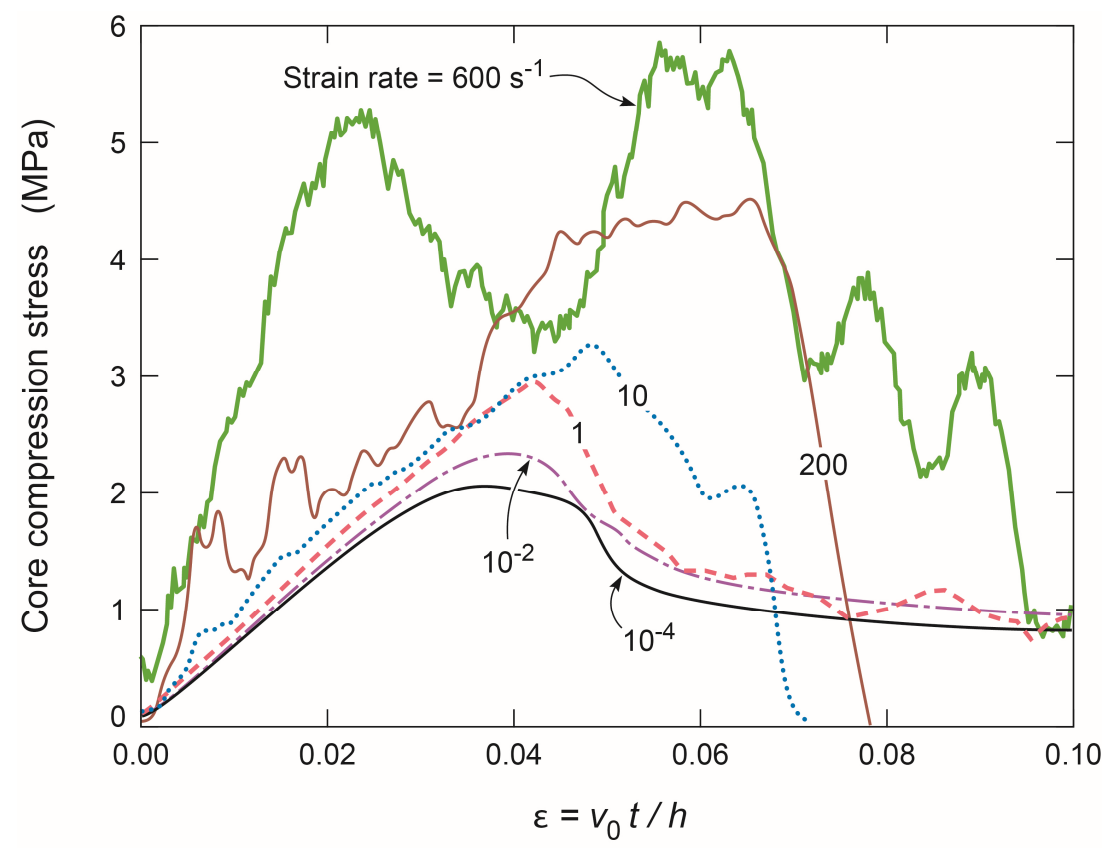

Figure 9: The nominal compressive stress versus strain response of the intermediate corrugated core configuration.

Recall that the tests on solid SrPET material in Fig. 4 illustrate that the plastic tangent modulus of the material increases from $E_{t}=2.2 \mathrm{GPa}$ at a strain rate of $10^{-4} \mathrm{~s}^{-1}$ to $4.4 \mathrm{GPa}$ at $\dot{\varepsilon}=1 \mathrm{~s}^{-1}$. This increase in $E_{t}$ results in an increase in the collapse strength of the corrugated core from 2.1 $\mathrm{MPa}$ to about $3 \mathrm{MPa}$ as the applied strain rate is increased to $1 \mathrm{~s}^{-1}$. There is no significant change in the initial elastic modulus of the corrugated core in this regime.

A further increase in the applied strain rate to $\dot{\varepsilon}=200 \mathrm{~s}^{-1}$ now results in an increase in the initial stiffness of the corrugated core and a very significant increase in the collapse strength as seen in Fig. 9. This increase is due to micro-inertial stabilisation of the core struts against plastic buckling and the corrugated core now attains a peak strength of about $5 \mathrm{MPa}$. A further increase in the applied strain rate to $600 \mathrm{~s}^{-1}$ does not change the peak strength significantly. Substituting $\sigma=5 \mathrm{MPa}$ in Eq. (2) gives a strut stress $\sigma_{c m} \approx 130 \mathrm{MPa}$ which is near the ultimate compressive strength of the SrPET solid material. This is confirmed via the high-speed photographs included in Fig. 10 which show the material failure near in the middle of the struts for both the $\dot{\varepsilon}=200 \mathrm{~s}^{-1}$ and $600 \mathrm{~s}^{-1}$ cases. We thus argue that micro-inertial stabilisation results in plastic buckling being suppressed to a point, that the stress in the core struts reaches the ultimate compressive strength of the solid material whereby the collapse mechanism now 
switches to material failure. Dynamic out-of-plane compressive response of stubby core configuration

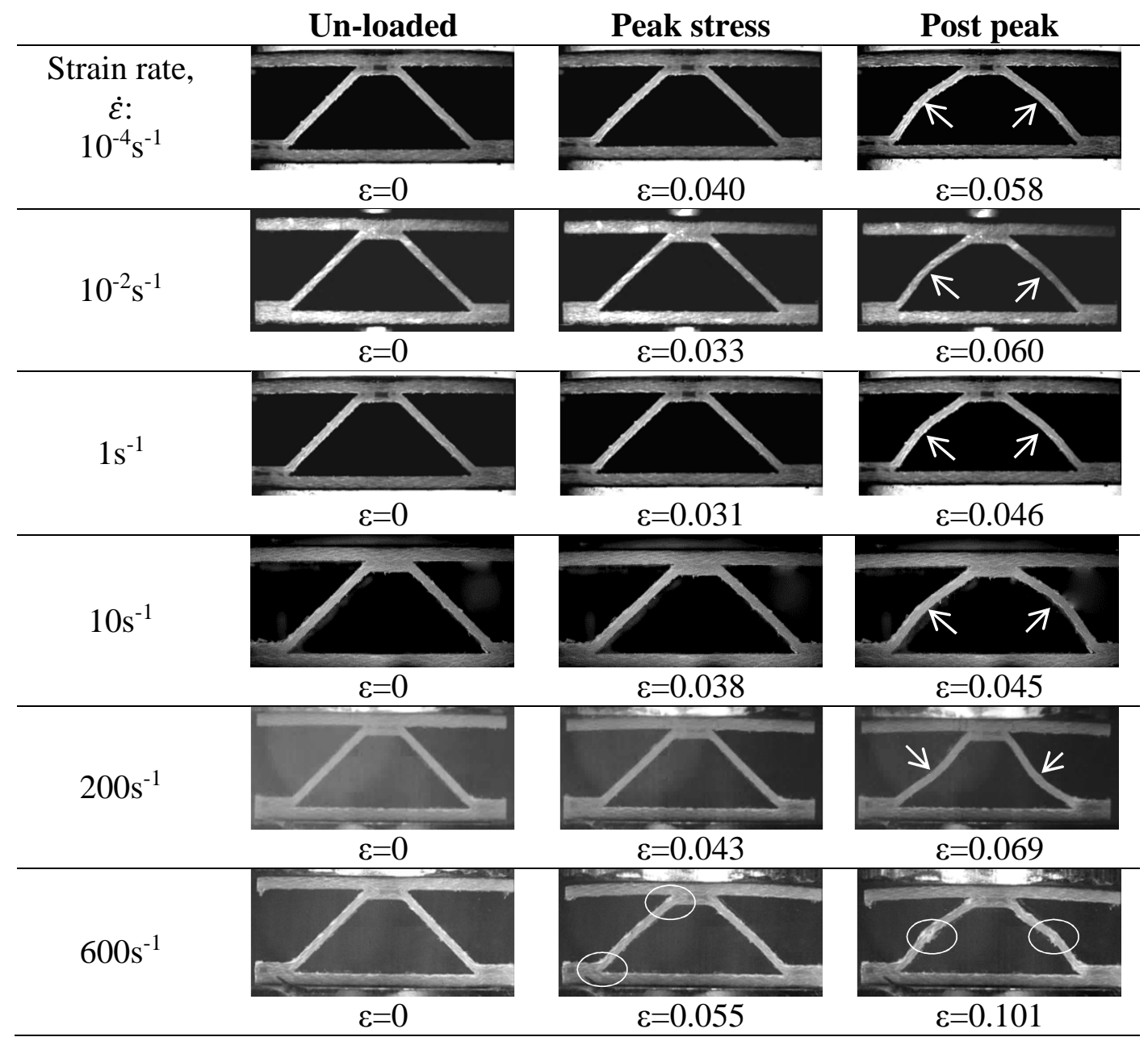

Figure 10: Montage of photographs showing the compressive deformation of the intermediate corrugated core configuration at three levels of compressive strain $\varepsilon$ for selected values of the applied strain rate $\dot{\varepsilon}$ ranging from quasi-static to $\dot{\varepsilon}=600 \mathrm{~s}^{-1}$.

The analysis of Section 5.1 predicts that the collapse strength of the stubby corrugated core configuration under quasi-static loading conditions is $5.9 \mathrm{MPa}$ if the collapse mode is plastic buckling of the struts and 6.1 MPa if compressive failure of the solid material is the operative collapse mechanism. The measured $\sigma$ versus $\varepsilon$ responses for the stubby configuration are included in Fig. 11. Again an initial elastic response is observed followed by a distinct peak stress under the quasi-static loading conditions $\left(10^{-4} \mathrm{~S}^{-1} \leq \dot{\varepsilon} \leq 10^{-2} \mathrm{~s}^{-1}\right)$. The measured peak strengths for both $\dot{\varepsilon}=10^{-4} \mathrm{~s}^{-1}$ and $10^{-2} \mathrm{~s}^{-1}$ are slightly lower than the predicted values: with the collapse predicted to occur at the transition between two regimes it is anticipated that the response is strongly imperfection sensitive [16] and this explains the over-prediction by the analytical estimates of Section 5.1. In fact, photographs showing the progression of deformation 
of the stubby core in Fig. 12 clearly show that while plastic buckling is the operative collapse mechanism at $\dot{\varepsilon}=10^{-4} \mathrm{~s}^{-1}$, compressive failure of the solid material occurs at $\dot{\varepsilon}=10^{-2} \mathrm{~s}^{-1}$.

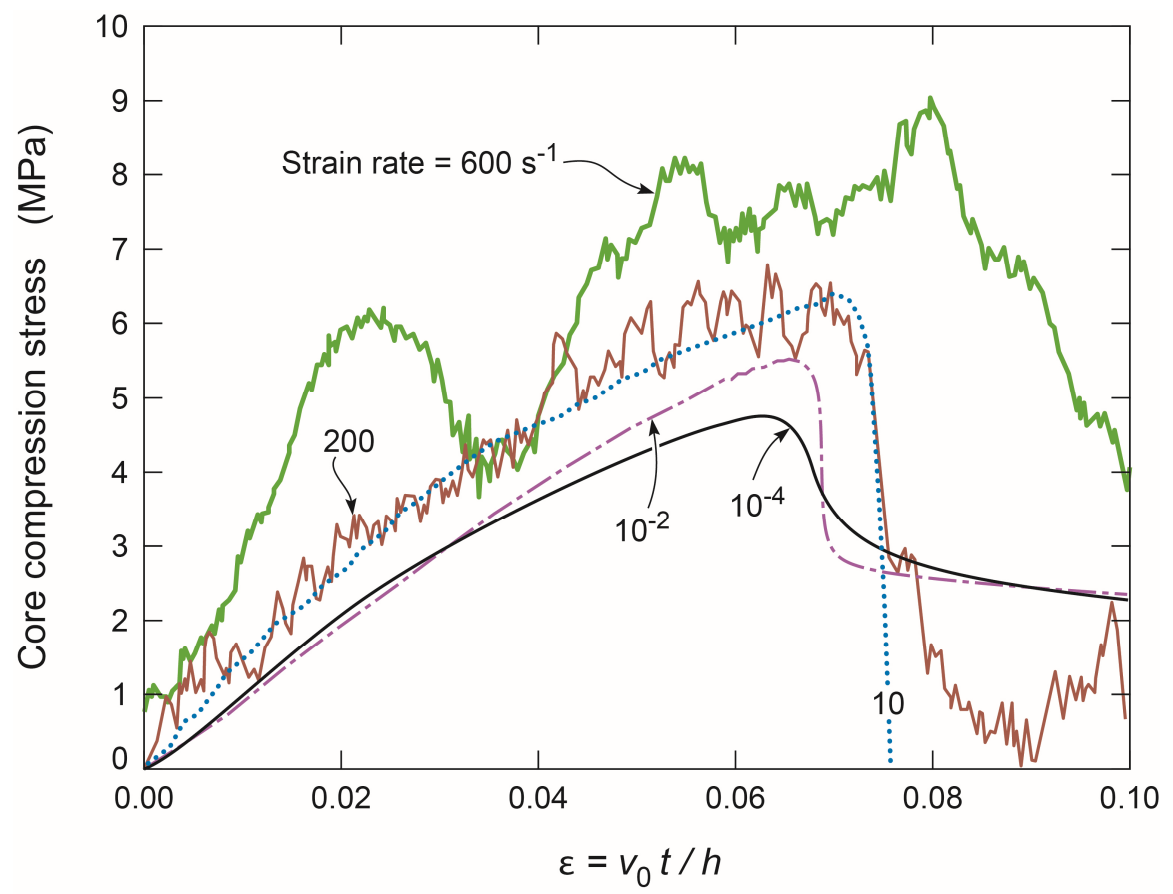

Figure 11: The nominal compressive stress versus strain response of the stubby corrugated core configuration.

With compressive material failure already being operative at $\dot{\varepsilon}=10^{-2} \mathrm{~s}^{-1}$, further increasing the applied strain rate does not change the collapse strength of the corrugated core significantly as seen in Fig. 11. This is also confirmed from the images showing the deformation of the core in Fig. 12 which clearly demonstrate that compressive failure of the material remains the operative collapse mode even for the highest value of $\dot{\varepsilon}$ investigated here. It is however worth emphasizing that in line with the increase in the modulus of the solid material with increasing strain rate the stiffness of the corrugated core is seen to increase as the strain rate is increase above $\dot{\varepsilon}=1 \mathrm{~s}^{-1}$. 


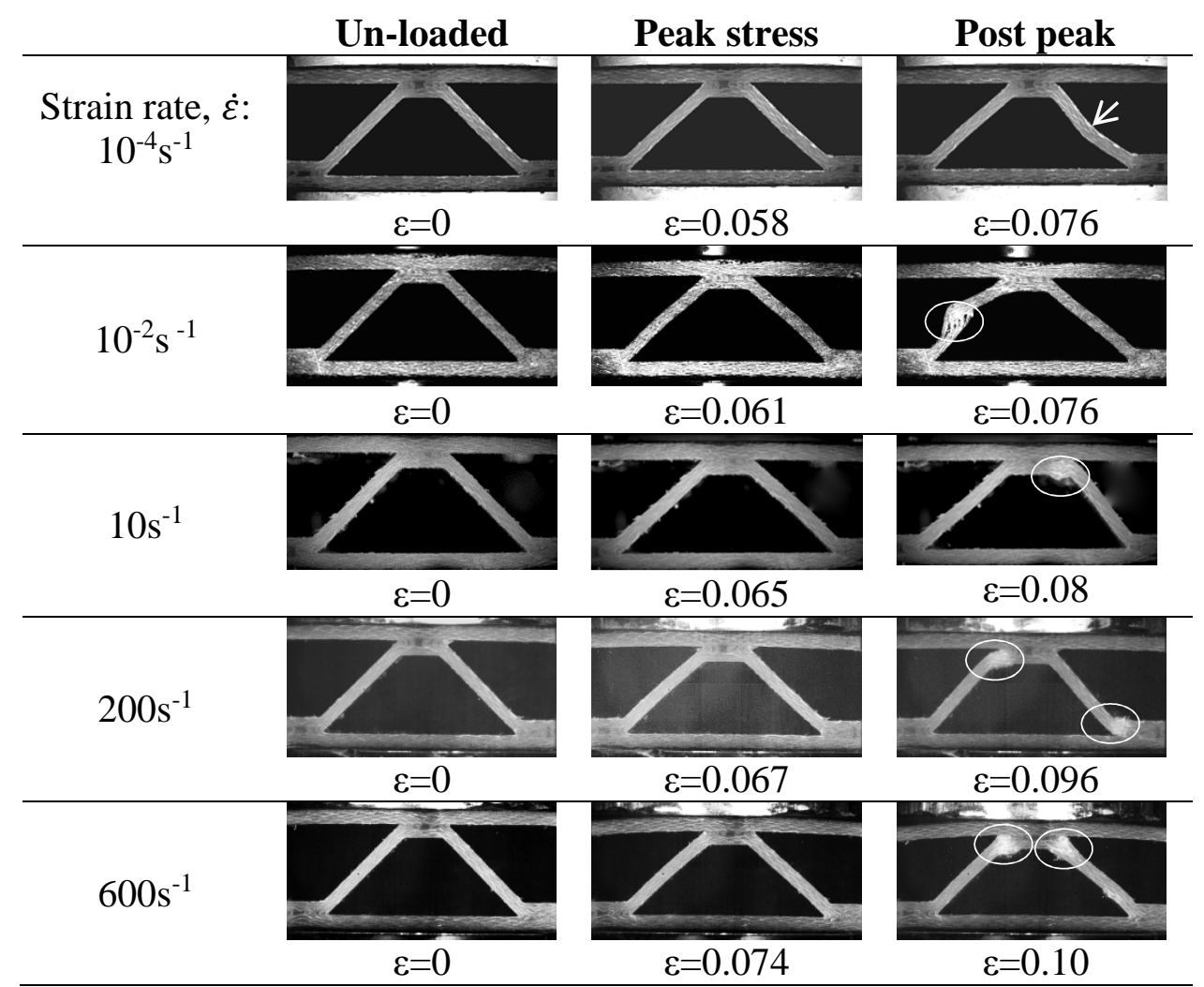

Figure 12: Montage of photographs showing the compressive deformation of the stubby corrugated core configuration at three levels of compressive strain $\varepsilon$ for selected values of the applied strain rate $\dot{\varepsilon}$ ranging from quasi-static to $\dot{\varepsilon}=600 \mathrm{~s}^{-1}$.

\subsection{Summary and discussion on failure modes}

The differences in the responses of the three corrugated core configurations investigated here can be summarised by comparing the core stress $\sigma_{c m}$ at peak load as a function of the applied strain rate $\dot{\varepsilon}$. We obtain an estimate of $\sigma_{c m}$ by substituting the measured peak value of $\sigma$ in Eq. (2) and then solving for $\sigma_{c m}$ using the geometrical parameters listed in Table 1. The deduced values of $\sigma_{c m}$ as a function of $\dot{\varepsilon}$ are plotted in Fig. 13 with $\sigma_{c m}$ normalised by the peak compressive strength $\hat{\sigma}$ of the solid SrPET material under the same nominal strain rate to only consider inertial stabilization. 


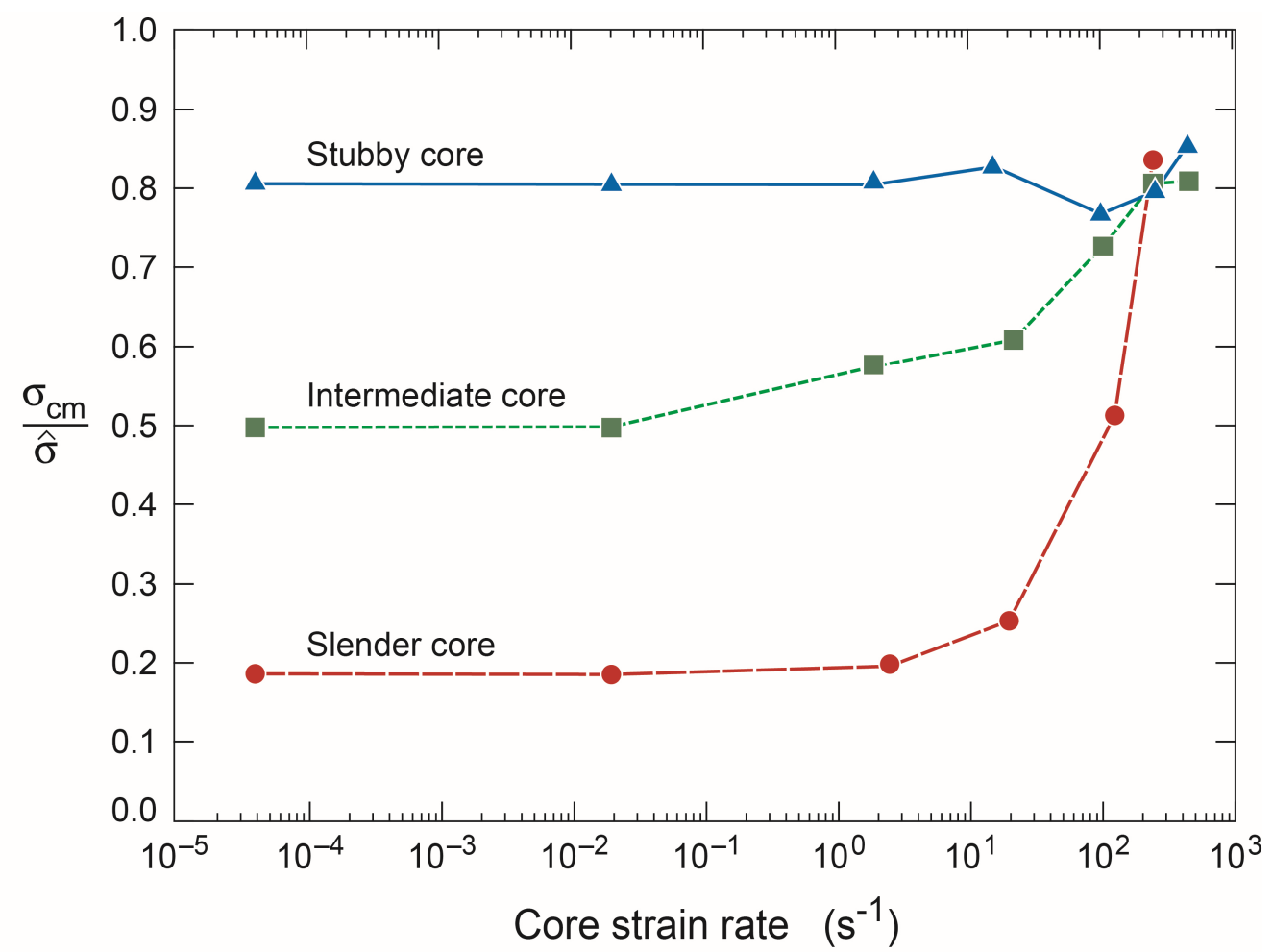

Figure 13: The measured core member stress $\sigma_{c m}$ at peak load normalized by the solid material peak compressive strength $\hat{\sigma}(\dot{\varepsilon})$ as function of the nominal corrugated core strain rate $\dot{\varepsilon}$.

First consider the case of the stubby core. The stress $\sigma_{c m}$ shows is approximately equal to $80 \%$ of the ultimate material strength. This is because the stubby core fails by compressive failure of the material over the whole range of strain rates investigated here with the $20 \%$ knockdown compared to the solid material strength attributed to imperfections in the as-manufactured corrugated core. Next consider the slender corrugated core. In this case $\sigma_{c m}$ displays a strong strain rate sensitivity. At low values of $\dot{\varepsilon}$, failure is by elastic buckling and hence $\sigma_{c m} / \hat{\sigma}$ is relatively small. However, micro-inertial stabilisation inhibits elastic buckling of the core struts for $\dot{\varepsilon}>1 \mathrm{~s}^{-1}$ and $\sigma_{c m} / \hat{\sigma}_{0}$ rises to approximately 0.85 at $\dot{\varepsilon}=600 \mathrm{~s}^{-1}$ with the collapse strength of the core nearly equal to that required for collapse to occur by the compressive failure of the solid material. The corrugated core with the intermediate value of the core strut aspect ratio collapse by plastic buckling of the core struts under quasi-static loading conditions and hence $\sigma_{c m} / \hat{\sigma}_{0}$ is higher at low values of $\dot{\varepsilon}$ in this case compared to the slender corrugated core. However, again with increasing strain rate micro-inertial stabilisation inhibits plastic buckling of the core struts and $\sigma_{c m} / \hat{\sigma}_{0}$ increases for $\dot{\varepsilon}>1 \mathrm{~s}^{-1}$ with collapse of the core occurring by compressive failure of the solid material for $\dot{\varepsilon} \geq 400 \mathrm{~s}^{-1}$.

\subsection{Comparison of the strain rate sensitivity of polymeric cellular structures}


Polymeric cellular structures and in particular polymer foams are known to be relatively strain rate insensitive (see for example the study of Tagarielli et al. [17] on PVC foams) with the rate sensitivity mainly due to the strain rate sensitivity of the solid polymeric material. The solid SrPET material employed in this study too has a relatively low strain rate sensitivity (yield strength increasing by approximately a factor of 2 over a strain rate range spanning 7 decades) but the corrugated cores with slender struts made from this material display a strong strain rate sensitivity. This sensitivity is attributed to micro-inertial effects. Calladine and English [14] investigated this phenomenon by examining two classes of structures, the so-called Type I and Type II structures. Type I structures have a "flat-topped" quasi-static stress versus strain curve and deform by the bending of their constituent struts similar to the deformation mechanism in foams. By contrast, the initial yield strength of Type II structures is set by the stretching of the constituent struts of the structure and typically display a softening compressive stress versus strain curve due to the buckling of these struts. In Type II structures, the buckling of the struts is stabilised by the inertia of the struts under high rates of loading (referred to as micro-inertial effects) while the bending-dominated response of the Type I structures is relatively inertia insensitive. The dynamic strength enhancements of the SrPET corrugated core are compared to a polymethacrylimide (PMI) Rohacell foam of similar density $\left(75 \mathrm{kgm}^{-3}\right.$ and $\left.200 \mathrm{kgm}^{-3}\right)$ [18, 19] in Fig. 14. Here $\hat{\sigma}_{D}$ is the ratio of the dynamic peak strength at a strain rate $\dot{\varepsilon}$ to the corresponding peak strength at the quasi-static strain rate $\dot{\varepsilon}_{0}$ where $\dot{\varepsilon}_{0}=10^{-4} \mathrm{~s}^{-1}$ and $10^{-3} \mathrm{~s}^{-1}$ for the corrugated cores and PMI foams, respectively. Both types of cellular structures are made from relatively rate-insensitive polymers and have similar strut aspect ratios as indicated in Fig. 14 but rather different topologies. The strut aspect ratio of the foam material corresponds to the ratio between the length and thickness of the cell wall edges as measured using Scanning Electron Microscopy $[18,19]$ The comparison clearly shows that for the same strut aspect ratio the corrugated core is significantly more rate sensitive compared to the PMI foam and this sensitivity increases with decreasing $t_{c} / \ell$ for the reasons discussed above. The comparison clearly shows the role of topology in governing the rate sensitivity of polymeric cellular materials. 


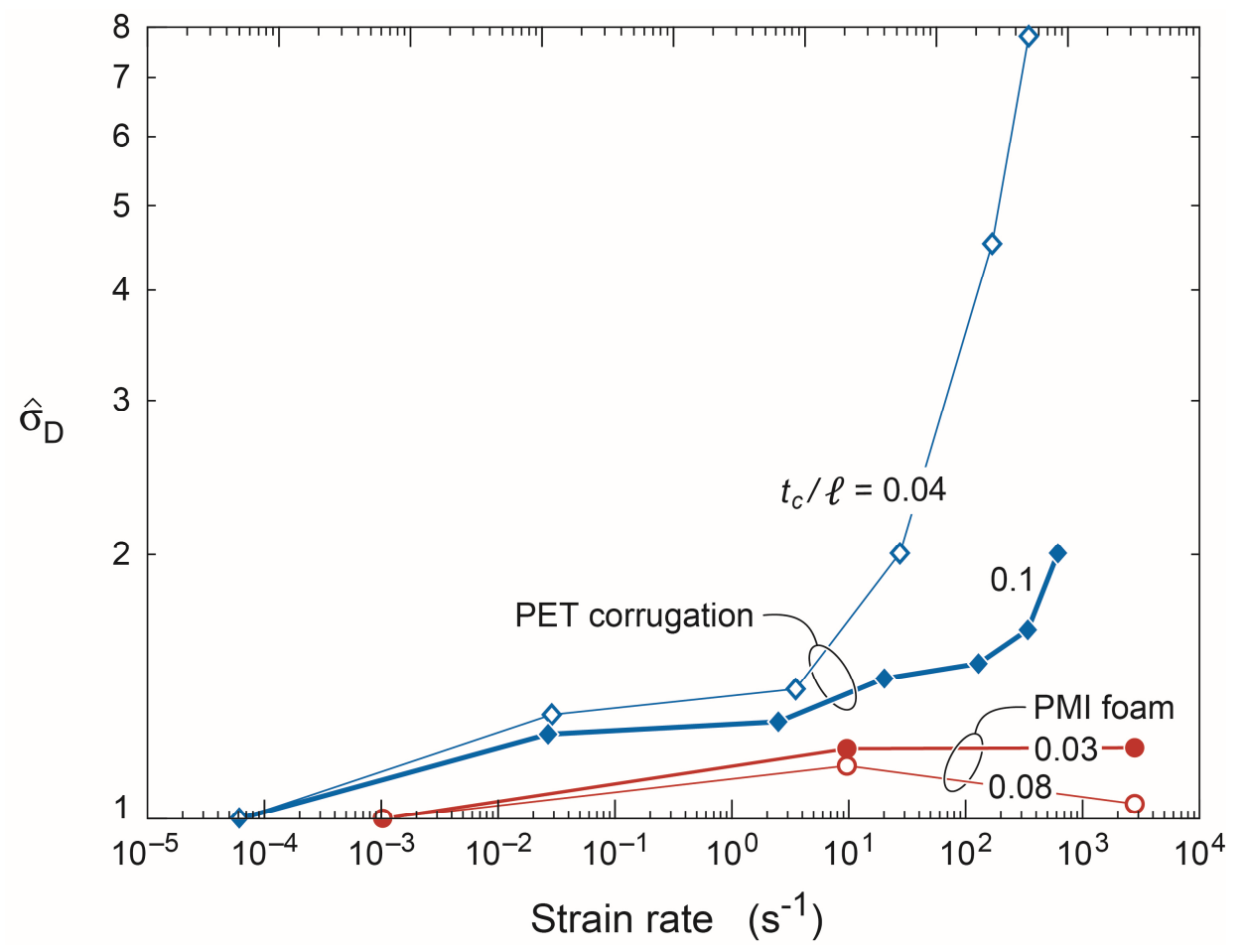

Figure 14: The dynamic peak strength enhancement $\hat{\sigma}_{D}$ as a function of the applied nominal strain rate $\dot{\varepsilon}$ for the SrPET corrugated cores and Rohacell PMI foams. Results are shown for two values of the strut aspect ratios $t_{c} / \ell$ in both cases. The foam slenderness ratios 0.03 and 0.08 are from foams with a density of $71 \mathrm{kgm}^{-3}$ and $200 \mathrm{kgm}^{-3}$ respectively.

\section{Concluding remarks}

Fully recyclable corrugated sandwich structures have been developed and the high strain rate compressive properties of both the parent material and the sandwich cores have been investigated. The parent solid material shows limited increase in strength and stiffness as function of strain rate but a significant increase in the material yield strength is observed with increasing strain rate.

Three different sandwich core densities have been investigated by varying the aspect ratio of the struts of the corrugated cores. The low density corrugated cores with slender constituent struts show significant strengthening with increasing loading rate while the higher density cores display only a limited rate sensitivity. The significant strengthening of the low density cores is mainly caused by micro-inertial stabilization of the core members against buckling. These micro-inertial effects do not play a significant role in typical polymeric foams which deform by the bending of the cell walls and thus these foams display limited rate sensitivity. The study has clearly highlighted the role of topology in governing the strain rate sensitivity of polymeric cellular materials. 


\section{Acknowledgements}

We thank for the financial support for this investigation from the Centre of ECO2 Vehicle Design and the Swedish Agency for Innovation Systems (VINNOVA). S. Kazemahvazi and V.S. Deshpande acknowledge Defence Advanced Research Projects Agency (Grant Number W91CRB-10-1-005) for the financial support for this research.

\section{References}

[1] Peijs T. Composites for recyclability. Mater. Today 2003;6:30-35.

[2] Karger-Kocsis J, Bárány T. Single-polymer composites (SPCs): Status and future trends. Compos Sci Technol 2014;94:77-94.

[3] Shivakumar Gouda PS, Vijay Chatterjee, Bahari PK, Dayananda Jawali, Sameer Rahatekar, Wisnom MR. Improving fracture toughness in carbon fibre epoxy composite through novel pre-preg coating method using Epoxy Terminated Butadiene Nitrile rubber. Mater Design 2014;62:320-326.

[4] Schneider C. Kazemahvazi S, Åkermo M, Zenkert D. Compression and tensile properties of self-reinforeced poly(ethylene terephthalate)-composites. Polym Test 2013;32:221-230.

[5] Alcock B, Peijs T. Technology and Development of Self-Reinforced Polymer Composites. Adv Polym Sci 2013;251:1-76.

[6] Schneider C., Velea M.N, Kazemahvazi S, Zenkert D. Compression properties of novel thermoplastic carbon fibre and PET fibre composite lattice structures. Mater Design 2015;65:1110-1120.

[7] Cabrera NO, Aclock B and Peijs T. Design and manufacture of all-PP sandwich panels based on co-extruded polypropylene tapes. Compos: Part B-Eng, 2008;39:1183-1195

[8] Comfil ApS. Thermoplastic Composites, DK 8883 Gjer, Denmark, 01.2015, www.comfil.biz

[9] GOM Gmbh. User Manual: Aramis v5.3.0, Braunschweig, Germany http://www.gom.com

[10] Kazemahvazi S, Russel B.P, Zenkert D. Impact of carbon fibre/epoxy corrugated cores. Compos Struct 2012;94:3300-3308.

[11] Mulliken AD, Boyce MC. Mechanics of the rate-dependent elastic-plastic deformation of glassy polymers from low to high strain rates. Int J Solids Struct 2006;43:1331-1356 
[12] Richeton J, Ahzi S, Vecchio KS, Jiang FC, Adharapurapu RR. Influence of temperature and strain rate on the mechanical behavior of three amorphous polymers: Characterization and modeling of the compressive yield stress. Int J Solids Struct 2006;43:2318 - 2335 .

[13] Coté F, Deshpande VS, Fleck NA, Evans AG. The compressive and shear responses of corrugated and diamond lattice materials. Int J Solid Struct 2006;43:6220-42

[14] Calladine CR, English RW. Int J Mech Sci 1984;26:689-701.

[15] Tilbrook, M.T., Radford, D.D., Deshpande, V.S., Fleck, N.A. Dynamic crushing of sandwich panels with prismatic lattice cores. Int J Solids Struct, 2007;44 :6101-6123.

[16] Hutchinson JW, On the postbuckling behavior of imperfection- sensitive structures in the plastic range, ASME Pap 71-APM 1971.

[17] Tagarielli VL, Deshpande VS, Fleck NA, The high strain rate response of PVC foams and end-grain balsa wood, Compos: Part B-Eng, 2008;39:83-91

[18] Arezoo S, Tagarielli VL, Petrinic N and Reed JM, The mechanical response of Rohacell foams at different length scales. J Mater Sci, 2011;46:6863-6870

[19] Arezoo S, Tagarielli VL, Siviour CR and Petrinic N. Compressive deformation of Rohacell foam: Effects of strain rate and temperature. Inter J Impact Eng 2013;51:5057. 